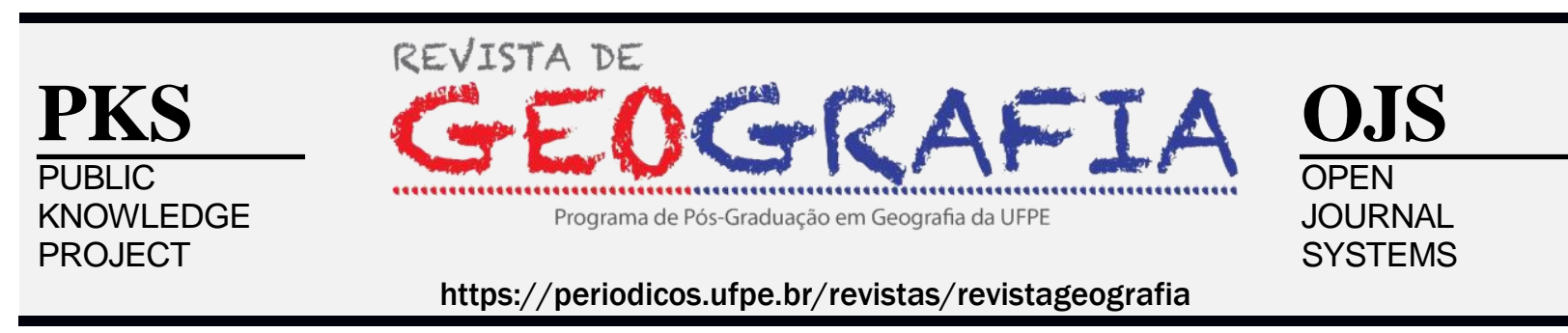

\title{
CONSIDERAÇÕES SOBRE A RELAÇÃO DO URBANO COM A CULTURA
}

Filipe Gomes Paulo ${ }^{1}$

${ }^{1}$ PPGG/UFRJ. E-mail: filipegeouerj@gmail.com

Artigo recebido em 15/06/2017 e aceito em 02/03/2020

\begin{abstract}
RESUMO
Embora haja um longo conhecimento de estudos urbanos pelos geógrafos, a incorporação do urbano pela Geografia Cultural foi tardia, a partir de meados da década de 1970. O urbano começa a ser foco de interesse por parte dos geógrafos humanistas e dos culturais que participaram da coletânea organizada por Agnew, Mercer e Sopher (1984) sobre a cidade nos diversos contextos culturais. O autor apresenta brevemente algumas análises feitas na perspectiva da Geografia Cultural, toponímia, a produção de formas simbólicas e a paisagem urbana. Neste artigo outros estudos são apresentados, sabendo-se que não se esgotam as possibilidades de abordar o urbano na perspectiva da Geografia Cultural.

Palavras-chave: cultura, urbano, contextos.
\end{abstract}

\section{CONSIDERATIONS ON THE URBAN RELATIONSHIP WITH CULTURE}

\begin{abstract}
There was a long knowledge of urban studies by geographers, an incorporation of the urban by Cultural Geography was delayed, from the mid-1970s. Gold begins to be a focus of interest on the part of the humanist and cultural geographers who participate in Da A collection organized by Agnew, Mercer and Sopher (1984) on the city in different cultural contexts. The author briefly presents some analyzes in the perspective of Cultural Geography, toponymy, a production of symbolic forms and an urban landscape. In this article, other studies are presented, knowing that they are not exhausted as possibilities to approach the urban in the perspective of Cultural Geography.
\end{abstract} Key words: culture, urban, contexts. 


\section{Introdução}

A representação de um dado aspecto da realidade implica a seleção de alguns de seus atributos e um jogo comparativo entre aquilo que se pensa conhecer e aquilo que não se conhece e é o objeto da representação. Neste processo diversas estratégias de retórica são adotadas, como as figuras de linguagem a exemplo dos tropos - metáforas, metonímias, sinédoques e alegorias - e das figuras de pensamento como a ironia (Duncan, 1996). Os tropos fazem parte da linguagem do cotidiano e da linguagem científica. Por meio deles e influenciado pela imaginação criativa os significados são construídos e reconstruídos (Cosgrove, 2000). Os significados são marcados pela instabilidade.

As representações sobre a natureza e o espaço socialmente construído incluem inúmeras figuras de linguagem como já apontado por Berdoulay (1982) a propósito das metáforas organicistas na linguagem dos geógrafos franceses, e por Livingstone e Harrison (1982), que também apontam a importância da visão organicista na elaboração de conexões entre metáforas, mitos e modelos. Barnes (1992), por sua vez, comenta o emprego de metáforas provenientes da Física na Geografia Econômica de matriz neo-clássica e da Biologia quando calcada na visão marxista: exemplificam-se, respectivamente, com os princípios de conservação de energia e da reprodução humana. Também a paisagem cultural é descrita por meio de metáforas como texto (Duncan, 1990) e espetáculo e texto (Daniels e Cosgrove, 1993), enquanto a própria cultura foi interpretada como mapas de significados, uma metáfora impregnada da dimensão espacial (Jackson, 1989).

Os estudos sobre o urbano, particularmente sobre a cidade, também estão impregnados de metáforas, metonímias e sinédoques, revelando que a cidade está sendo interpretada como um texto. Neste sentido, o livro de Duncan (1990) sobre a paisagem urbana da capital do reino de Kandy no Sri Lanka das primeiras décadas do século XIX é notável: o segundo capítulo, ‘A Paisagem como um Sistema de Criação de Signos', de natureza teórico-metodológica, foi recentemente publicado em português (Duncan, 2004).

O emprego de tropos representa uma interpretação passível de re-interpretação. Assim, a "cidade informacional" de Castells (1989) é uma metáfora que descreve aquelas cidades que concentram as denominadas atividades quaternárias, inclusive, e sobretudo, aquelas de decisão e controle de atividades localizadas em inúmeros lugares. Informações fluem de e para essas cidades, envolvendo numerosos lugares. A natureza dessas informações varia ao se considerar aquelas que fluem descendentemente, ordens, e ascendentemente, avaliações e informações em 
geral que alimentam as decisões e o controle por parte da "cidade informacional". Trata-se de uma metáfora tecnológica, possível apenas no último quartel do século XX. Contudo, é possível ver nesta metáfora uma interpretação que esconde o papel dessas cidades como foco principal de acumulação de capital, cuja ação interfere em amplos espaços, remodelando-os e controlando-os, e sobre milhões de pessoas. Afinal, é das "cidades informacionais" que (re)flui capital para fora e retornam lucros para as grandes corporações nelas sediadas.

O emprego dos tropos como meio de análise crítica de estudos urbanos é exemplificado com o artigo de Duncan (1996). Sua referência básica é a contribuição de Hayden White, historiador que nas décadas de 1970 e 1980 utilizou-se dos tropos na análise do discurso histórico (Duncan, 1996). Duncan analisa três autores em suas tentativas de descrever a cidade moderna. Seu interesse não reside em julgar os méritos dos trabalhos analisados, mas em ver como cada um deles descreve a cidade moderna. São assim analisados o clássico artigo de Ernest W. Burgess, publicado em 1925, "The Growth of the City - An Introduction to a Research Project", o livro de Mike Davis, publicado em 1992, Fortress Los Angeles: The Militarization of Urban Space, e o livro de Roland Barthes, tradução inglesa de 1982, Empire of Signs, que analisa as cidades japonesas. Cada um dos autores emprega, segundo Duncan, três tropos, respectivamente, metáfora, sinédoque e ironia.

A influência das ciências naturais na Escola de Chicago, da qual Burgess foi um dos expoentes, traduziu-se, em parte, pelo uso da linguagem das ciências naturais, caracterizada pela pretensa objetividade e capacidade de dar inteligibilidade à organização espacial e dinâmica da cidade. Seu texto, argumenta Duncan, tem como principal figura de linguagem a metáfora da cidade como um organismo vivo. Seu "crescimento é visto como resultante da organização e desorganização análogos aos processos anabólicos e catabólicos do metabolismo do corpo" humano (Duncan, 1996, p. 257). Assim, por exemplo, a massiva imigração de negros provenientes do sul dos Estados Unidos para Chicago, após a Primeira Guerra Mundial, é interpretada como um distúrbio no metabolismo da cidade.

A mobilidade foi considerada o "pulso da comunidade", e esta, em muitos casos, vista analogamente à ecologia vegetal. Deste modo, o processo de mudança do conteúdo social de áreas residenciais, via de regra gerando uma diminuição do status social da área, foi definido como de invasão-sucessão, uma metáfora biológica, análogo ao das comunidades vegetais. Duncan argumenta ainda que o próprio esquema gráfico pode ser visto como uma metáfora. Considerado clássico na literatura sobre o urbano, esse esquema descreve cinco zonas organizadas espacialmente em círculos concêntricos, o mais central deles referindo-se ao centro 
da cidade; há um aumento do status social à medida que se passa de um círculo para outro, do centro para a periferia. Este esquema representa a projeção "de um tipo de organismo cuja estrutura é visível para o cientista (...) através de um microscópio” (Duncan, 1996, p. 258).

O texto de Mike Davis, por outro lado, está calcado em uma leitura marxista da sociedade capitalista. Duncan argumenta que os aspectos perversos que Davis aponta "não são inerentes à cidade em si mesma, mas à brutalidade da vida sob o capitalismo, após o fim do liberalismo" (Duncan, 1996, p. 259). A figura de linguagem contida no texto de Davis é a sinédoque, na qual a cidade é considerada uma parte essencial do todo, o capitalismo. Deste modo, "a cidade expressa o capitalismo e seus problemas devem ser entendidos como idênticos aos problemas mais amplos do capitalismo" (Duncan, 1996, p. 259). De acordo com Duncan, a história que Davis nos conta sobre a auto-segregação, os guetos e barrios, a invasão dos espaços públicos e a repressão, enquadra-a dentro do modo trágico de explicação. Trata-se de uma análise influenciada pelo pensamento marxista, profundamente distinta daquela realizada por Burgess.

A obra de Roland Barthes, finalmente, privilegia, em princípio, a cidade de Tóquio. Em realidade, contudo, o autor, segundo Duncan, está mais interessado em evidenciar a natureza muito problemática das representações, tentando derrubar a normalmente aceita relação entre aqueles que estabelecem os significados e estes. Deste modo, Barthes adota como figura de linguagem básica a ironia, que implica não ter nada a dizer sobre Tóquio, pois toda a possibilidade de representação foi eliminada.

Duncan argumenta em seu Me(tropo)le que nos três textos a cidade é diferentemente considerada e, por isso, os três autores adotaram distintas figuras de linguagem. As representações "ligam os significados dos textos intertextualmente às tradições intelectuais, ciência natural, marxismo e crítica literária pós-estruturalista” de seus autores (Duncan, 1996, p. 264).

A contribuição de James Duncan abre perspectivas interessantes para a Geografia brasileira. Quais figuras de linguagem estão subjacentes à produção da Geografia, urbana ou não, brasileira? A este respeito sugere-se a possibilidade de ser incorporada a proposição de Hayden White, influenciada pelos críticos literários N. Frye e K. Burke, tal como apresentada por Smith (1996). Nesta proposição argumenta-se que um texto geográfico pode apresentar-se segundo quatro modos ficcionais: romance, comédia, tragédia e ironia. Quatro figuras de linguagem, por outro lado, constituem as representações que os geógrafos constroem sobre a 
realidade: metáforas, metonímias, sinédoques e ironia. Esta perspectiva possibilita avaliar a produção geográfica brasileira e ampliar nosso conhecimento sobre essa mesma realidade.

\section{A Identidade da Cidade como Construção Cultural}

A identidade de uma cidade, vista como lugar dotado de uma singularidade que o distingue dos outros, pode ser de modo marcante o resultado de construção intelectual, derivada de uma tradição étnica cujos traços são permanentemente recriados. Esta identidade cultural do lugar, reconhecida tanto interna quanto externamente (Relph, 1976), expressa-se em vários aspectos relativos à sua organização social, política e espacial. Esta identidade, no entanto, pode ser objeto de contestação por meio de práticas políticas que introduzem novos significados ao lugar, criando um confronto identitário. O exemplo de San Antonio, no Estado norte-americano do Texas, é a este respeito muito pertinente, como se depreende dos textos de Areola (1994) e Oliver (1996). Ali existe uma tensão de identidades, que tem suas origens e dinâmica na história local e do território texano.

A cidade de San Antonio tem sua identidade definida por ser a capital cultural mexicana nos Estados Unidos, embora em Los Angeles a população etnicamente mexicana seja numerosamente mais importante. Entretanto, em San Antonio cerca de 50\% da população é de origem mexicana, enquanto em Loa Angeles este valor cai para 20\% (Areola, 1994).

A origem de San Antonio remonta ao começo do século XVIII. Em 1770 passou a ser a capital da Província do Texas, na Nova Espanha, colônia espanhola. Em 1811 é elevada à categoria de cidade, e em 1821 parte da República do México. Em 1836 a cidade passa a fazer parte da efêmera República do Texas, e em 1845 parte do Estado do Texas, que adere à federação norte-americana. A mudança política foi acompanhada por mudanças na composição da população da cidade: em 1835 toda a população de San Antonio era de nacionalidade mexicana, mas em 1850 a população de origem mexicana representava $46 \%$ do total, graças à imigração de americanos e europeus.

Beneficiada nas décadas de 1870 e 1880 pelas ferrovias que a ligavam ao interior do território americano e às principais áreas mexicanas, a cidade de San Antonio tornou-se o foco principal de correntes migratórias procedentes do México, assim como o ponto de redistribuição de mão-de-obra para as atividades agrícolas de ampla área dos Estados Unidos. A cidade tornase, a partir do começo do século XX, o foco dispersor de traços culturais mexicanos como a comida e a música (criação do estilo musical denominado 'conjunto'). Torna-se, ainda, o foco 
político para os descendentes mexicanos, a partir de instituições ligadas aos direitos civis e ao 'La Raza Unida Party’, partido político criado em 1970, que tem seções em todo o Sudoeste norte-americano. São os texanos de origem mexicana que controlam instituições municipais como a vinculada à educação. Por volta de 1990, o prefeito e o bispo de San Antonio eram de origem mexicana.

A força da comunidade de origem mexicana levou em 1944 à instalação na cidade de um 'campus avançado' da UNAM (Universidad Nacional Autonoma de Mexico), a mais importante universidade mexicana. Por sua vez, em uma cidade profundamente segregada do ponto de vista étnico, os bairros constituídos por texanos de origem mexicana estão organizados, com base nas paróquias católicas, em comunidades que atuam na reivindicação de solução para problemas como saneamento e transporte (Areola, 1994).

À San Antonio de raízes e traços mexicanos contrapõe-se uma outra San Antonio, de traços norte-americanos. À identidade de raízes mexicanas estabelece-se outra identidade, criada mais tardiamente, dotada, contudo, de força, pois identifica a cidade em escala nacional. Trata-se, portanto, de identidades distintas, que coexistem na mesma cidade.

Na periferia de San Antonio está o forte do Alamo, parte integrante dos sangrentos episódios que levaram à incorporação do Texas e da cidade de San Antonio aos Estados Unidos durante a década de 1830. O forte era originalmente uma missão católica espanhola, mas ali travaram-se, em 1836, intensos combates entre soldados americanos e mexicanos. Os primeiros lutavam contra a tirania do ditador mexicano General Santa Anna, e os mexicanos contra a perda do território texano, ocupado progressivamente pelos criadores de gado procedentes do território americano. A derrota dos americanos neste episódio foi transformada em um feito histórico, simbolizando a luta do povo americano pela liberdade e direitos cívicos e contra a opressão (Oliver, 1999).

Abandonadas por quase 70 anos, as ruínas da antiga missão religiosa foram adquiridas em 1905 por um grupo de mulheres, Daughters of the Republic of Texas, que em vez de preservarem a missão, transformaram-na em local cívico, que celebraria o martírio daqueles americanos que ali morreram em nome de um ideal do povo americano. Altera-se o significado original da missão e, simultaneamente, altera-se sua forma e a área em torno. A capela, que ficava no interior da missão, é, por meio da derrubada dos muros, tornada visível e o ponto focal do novo Alamo; seu estilo barroco espanhol é adulterado; a capela é transformada em museu, com símbolos que se reportam aos 'mártires' americanos, negando-se a exibir qualquer objeto que se reportasse às culturas espanhola e indígena; e onde não havia nada, constrói-se 
um jardim à européia. Por sua vez, as ruas em torno do Alamo têm seus nomes alterados, designando os americanos mortos em 1836.

O Alamo transforma-se, assim, na principal fonte de atração turística de San Antonio. Em torno do agora renomeado forte Alamo estabelece-se importante área comercial com dois shopping centers, área de lazer ao longo do rio San Antonio e um canal artificial ligando os dois centros de compra. Transforma-se, assim, a intenção de se estabelecer um centro de peregrinação cívica. O Alamo torna-se um foco de peregrinação para o consumo. Mais do que isto, argumenta Oliver (1996), o Alamo transforma-se em foco de peregrinação cultural, pois ele e suas extensões comerciais estão encravados em área predominantemente de cultura mexicana ('latina').

Estabelece-se, assim, uma nítida diferenciação identitária. De um lado, uma cidade marcada por uma identidade mexicana, habitada por pessoas com nível de renda inferior e consideradas inferiores. De outro, estabelece-se um marco identitário de cultura dominante, opulenta e auto-considerada superior. O Alamo metamorfoseado é o símbolo dessa identidade.

A cidade de San Antonio tem, assim, uma dupla identidade, que se justapõe espacialmente, que apresenta temporalidades que parcialmente se recobrem, mas que compõem uma mesma unidade espacial e refletem um complexo processo de relações culturais, tensas e dramáticas.

\section{Verticalização: Formas Simbólicas e Representações}

A verticalização de expressiva área do espaço urbano constitui marcante traço da paisagem da cidade moderna (Relph, 1987), tendo suscitado inúmeros estudos. No Brasil, menciona-se, entre outros, os estudos de Spósito (1991), Souza (1994), Ramires (1998), Costa (2000) e Silva (2002 e 2004).

Segundo Domosh (1994), a verticalização pode ser estudada considerando a distribuição espacial dos prédios e suas relações com o preço da terra e amenidades, entre outros aspectos, suas relações com processos sociais em escala local e regional, como sendo o resultado de práticas sociais, envolvendo, entre outros, os promotores imobiliários, e como sendo um símbolo para o qual procura-se decodificar seu significado. Neste caso a verticalização deriva de uma ação simbólica por parte de promotores imobiliários que procuram enviar mensagens a respeito de prestígio, poder e legitimação para uma clientela, por meio de objetos estéticos, ou por parte dos usuários, associada à posse de um símbolo social. 
A cidade de New York constitui-se no melhor exemplo de adoção, ainda nas últimas décadas do século XIX, desse símbolo (Domosh, 1994). Entre 1850 e 1870 a elite mercantil de New York, a principal cidade comercial, industrial e portuária dos Estados Unidos, exibia seu poder e prestígio por meio de suntuosos palácios em estilo europeu, localizados ao longo da Broadway. A partir de 1870 começaram a surgir os primeiros edifícios altos, viabilizados em grande parte pela invenção e difusão do elevador. Esses edifícios foram construídos pelas próprias grandes empresas em expansão, ou com base em capitais especulativos. Entre essas empresas estavam as jornalísticas e de seguros em rápida expansão, acompanhando o crescimento econômico e demográfico da cidade. As empresas, que competiam entre si, vendiam uma imagem de poder, prestígio e estabilidade por intermédio de prédios monumentais, com torres, arcos, colunas e domos, em parte no estilo do Renascimento. Em 1875 o jornal New York Tribune ocupava um prédio com setenta e oito metros de altura. Em 1909 a empresa de seguros Metropolitan Insurance inaugurava seu prédio com altura equivalente a cinqüenta andares, dos quais quinze em forma de torre; possuía um grande relógio com quatro faces e no topo havia uma 'lanterna' iluminada à noite (Domosh, 1994).

Estudos realizados sobre cidades brasileiras também apontam a dimensão simbólica da verticalização, esta sendo, via de regra, parte integrante de análises focalizando diversos outros aspectos associados à produção e ao consumo de imóveis verticalizados. Símbolos de modernidade e status social, os prédios residenciais tendem, como exemplificado com Uberlândia (MG), estudada por Ramires (1998) e as cidades paulistas de Ribeirão Preto, São José do Rio Preto e Presidente Prudente (Spósito, 1991), a ratificar o valor simbólico, e de mercado, de áreas adjacentes ao núcleo central de negócios, áreas que em um passado recente eram habitadas pela população de alto status social. O sentido simbólico transparece nos folhetos de publicidade dos imóveis e nos nomes a eles conferidos, nomes de personalidades locais ou de expressão nacional ou internacional - proprietários fundiários, nobres, burgueses e intelectuais de prestígio, ou nome de lugares e objetos que denotam poder, prestígio e status social, como se exemplifica com os nomes de White House, Times Square, Grand Palais e Diamond Tower (Ramires, 1998).

O estudo de Silva (2002 e 2004) sobre a verticalização da cidade de Guarapuava, localizada nos campos do terceiro planalto paranaense e caracterizada por fortes vínculos com o mundo pastoril latifundiário, privilegia as representações sociais da elite local e de outros grupos sociais a respeito dos prédios altos, localizados, sobretudo, no centro da cidade. 
O estudo apóia-se no conceito de representações sociais de Sergio Mascovici, no qual essa é uma reconstrução da realidade, elaborada e re-elaborada por indivíduos e grupos sociais a partir de práticas e experiências. Pesquisa em dois jornais da cidade e por meio de entrevistas com moradores do centro, tanto de prédios verticalizados como de prédios térreos, mas ambos de alto status social, e com moradores da periferia, de baixo status social, permitiram identificar as representações sociais sobre a verticalização da cidade.

A pesquisa nos jornais possibilitou identificar que as representações sociais sobre a verticalização variaram ao longo do tempo, refletindo transformações da estrutura econômica, social e espacial da cidade. No período entre 1969 e 1979, a primeira fase da verticalização, as representações denotavam o sentido simbólico dos edifícios altos. Representavam eles um projeto coletivo da elite local, visando trazer o progresso à cidade e, ao mesmo tempo, enfatizavam o papel de liderança e empreendimento do grupo social e de cada um de seus membros. As palavras-chave das representações eram 'progresso', 'pessoalidade', 'guarapuavano', 'orgulho', 'proprietário' e 'luxo'.

No período entre 1986 e 1998 as representações denotavam outros sentidos. A cidade necessitaria competir com outros centros urbanos que apresentavam expressiva verticalização, denotando ainda a importância da diferenciação sócio-espacial e de auto-segregação. Nesse sentido, a altura dos prédios passa a ser mais importante que no período anterior. Associada à verticalização estão as empresas de fora, capazes de produzir prédios altos e projetar a cidade. Investimentos pela elite em prédios localizados no centro reafirmariam tanto o valor simbólico do centro, o lugar da elite, como a posição deste grupo social. Nesse sentido, as palavras-chave da representação são "altura", “empresa", “elite”, "localização", "pessoalidade” e "investimento".

A pesquisa com os três grupos sociais identificou representações dissonantes em relação à verticalização e sua localização predominantemente central, evidenciando ainda as representações a respeito das construções térreas tanto localizadas no centro como na periferia. Assim, por exemplo, a verticalização é interpretada pela elite que mora em prédios altos como sinônimo de ordem e riqueza; esse mesmo grupo considera a periferia local de desordem e pobreza. A periferia, por outro lado, vê o centro verticalizado como lugar sem natureza, violenta e insegura, a despeito de apresentar ordem.

O estudo de Silva aponta para as múltiplas representações a respeito da organização espacial da cidade, fragmentada, heterogênea e desigualmente vivenciada por uma população que, assim, reconstrói diferentemente a realidade. 


\section{Globalização e o Papel Cultural das Cidades Globais}

A despeito de certas lacunas do ponto de vista teórico, a literatura sobre a dimensão econômica das cidades globais é relativamente extensa e no plano descritivo já aponta alguns resultados consistentes. Ao contrário, muito mais escassa é a literatura sobre a dimensão cultural das cidades globais. O livro de Ulf Hannerz, Transnational Connections, aponta alguns aspectos, seguidos de exemplos, sobre essa dimensão (Hannerz, 1996).

Hannerz retoma a tese de Redfield e Singer sobre o papel das cidades na transformação cultural de suas hinterlândias. Publicada em 1954, os autores distinguem a cidade ortogenética, criação interna a uma dada cultura, como exemplificam as cidades cerimoniais da Antigüidade, da cidade heterogenética, criação de um grupo externo à região onde foi implantada, como exemplifica a cidade colonial. Enquanto o primeiro tipo de cidade re-elabora a cultura original, o segundo transforma-a profundamente. Com a globalização, argumenta Hannerz, emergem cidades que desempenham esse papel de transformação cultural de amplos espaços, constituindo cidades "proeminentemente engajadas em transformações e recombinações de significados e formas simbólicas, que estão alterando o mapa cultural da superfície terrestre" (Hannerz, 1996, p. 127).

Estas cidades desempenham esse papel de transformação cultural em ampla escala espacial por intermédio de quatro grupos sociais que nelas estão presentes, a saber:

1) Os empresários e executivos das empresas transnacionais, que trabalham em atividades "de direção, bancos e finanças, consultoria jurídica, contábil e técnica, telecomunicações e computação, transporte internacional, pesquisa e ensino de alto nível" (Hannerz, 1996, p. 129).

2) Os imigrantes provenientes dos denominados países do Terceiro Mundo, asiáticos, africanos, latino-americanos e caribenhos, que compõem parcela significativa dessas cidades globais, a exemplo de New York, Londres e Paris.

3) Pessoas diretamente vinculadas às atividades culturais strictu sensu, especializadas em atividades como "arte, moda, design, fotografia, cinema, literatura, música e cozinha" (Hannerz, 1996, p. 130). Muitos são estrangeiros.

4) Turistas nacionais e do exterior, que têm vínculo muito transitório com essas cidades globais. 
Hannerz argumenta que essas quatro categorias de pessoas estão articuladas entre si, seja no que diz respeito ao mercado de trabalho, seja no que se refere à esfera do consumo (por exemplo, migrantes e elite empresarial, especialistas em cultura e elite empresarial, turistas e especialistas em cultura). Em razão das interações entre essas quatro categorias de pessoas e também da mídia, estabelece-se um ambiente propício para transformar essas cidades globais em 'lugares centrais da cultura', onde são produzidas cultural commodities, sempre reinventadas e renovadas. Mas se as cidades globais são, de modo geral, os pontos iniciais dos fluxos culturais globais, são também postos de re-elaboração de aspectos da cultura externa, como a do Terceiro Mundo, os quais são, a seguir, reexportados (Hannerz, 1996).

As relações entre cidades globais da cultura e seus espaços são, assim, complexas. Essas cidades constituem focos de criação e de re-interpretação de significados e de formas simbólicas. Aponte-se que esse papel de transformação cultural está profundamente inserido no processo de acumulação de capital, sendo uma de suas expressões.

A globalização da cultura possibilitou a Hannerz (1996) elaborar o conceito de creole culture, um híbrido cultural. Segundo ele, este conceito envolve a combinação de diversidade, interconectividade e inovação no âmbito de complexas relações entre centro e periferia. A diversidade refere-se à confluência recente de tradições culturais histórica e geograficamente distintas, enquanto a interconectividade diz respeito às interações relativamente contínuas entre significados e formas simbólicas, gerando inovações mercantilizadas.

Se os países da periferia são profundamente impactados por significados e formas simbólicas provenientes dos países centrais, o mesmo se verifica em relação aos países centrais, particularmente suas metrópoles, a exemplo de Stockholm, analisada por Hannerz (1996) e Amsterdam, estudada por Nijman (1999).

A capital sueca apresenta características de uma cidade com fortes traços da creole culture, combinando aspectos culturais locais e originários de distintas áreas. Assim, no subúrbio de Botkyrka localizam-se conjuntos habitacionais com forte presença de imigrantes, entre eles turcos e do Oriente Médio. Isto introduz traços culturais distintos daqueles suecos, marcando a paisagem daquele subúrbio. Por outro lado, no centro de Stockholm a paisagem revela a forte presença de firmas estrangeiras, cujos nomes de ressonância internacional aparecem nas lojas e cartazes. A capital sueca é, assim, submetida a uma dupla creolization, como argumenta Hannerz. Em realidade, o processo de globalização cultural redefine as relações entre centro e periferia. Estes, centro e periferia, não podem mais ser definidos com 
base em uma necessária separação espacial. Centro e periferia e suas características são mutuamente influenciados por processos de incorporação e re-elaboração.

Amsterdam constitui exemplo dos efeitos da globalização cultural sobre a identidade de uma cidade. Em decorrência de seu papel como centro de turismo internacional, a cidade passa a ser vista como lugar de permissividade do sexo e do uso de drogas (Nijman, 1999).

Esta última imagem, contudo, está envolta em uma mistura de autenticidade e artificialidade. A globalização cultural, argumenta Nijman, tende a reforçar algumas características locais e a diluir outras. É o caso da identidade de Amsterdam, na qual foram minimizados traços identitários construídos desde o século XVII, enquanto outro foi reforçado, mas em outra direção. O calvinismo, o comercialismo, a frugalidade e a tolerância religiosa e quanto à nacionalidade, foram minimizados, enquanto a tolerância é reforçada no que diz respeito à liberdade sexual e ao uso de drogas.

A tolerância tornou-se uma marca, um selo identitário que permite à cidade ter seu nicho no mercado competitivo do turismo. Nas palavras de Nijman (1999, p. 155), "commodification of its identity as tolerant place".

Mas essa identidade é uma representação parcial da cidade, que mantém importante atividade comercial, agora não mais localizada em seu centro histórico, lugar do turismo, do sexo e das drogas, mas na periferia da cidade, em outro quadro físico, social e econômico, cuja importância não é ressaltada na imagem de cidade produzida e vendida internacionalmente.

A globalização tem sido abordada, sobretudo, na perspectiva econômica, incluindo, no caso do urbano, o estudo das cidades globais. A dimensão cultural da globalização, especialmente no que concerne às grandes cidades, necessita de mais estudos. Em relação ao Brasil, a lacuna parece ser ainda maior. Cidades como São Paulo, Rio de Janeiro, Salvador e Florianópolis, entre outras, merecem atenção dada as relações internacionais que apresentam.

\section{Considerações Finais}

O presente estudo apresentou algumas análises com que os geógrafos culturais abordam o urbano. As possibilidades são imensas na medida que não são temas que definem a análise cultural do urbano, mas um foco centrado na perspectiva dos significados. Isto possibilita que o urbano seja analisado segundo as mais diferentes óticas. O econômico, o político e o social, em sua espacialidade urbana, podem ser analisados na perspectiva da Geografia Cultural. Isto não nega as análises urbanas sob outras perspectivas. Ao contrário, reconhece seu valor e delas 
obtém informações a serem utilizadas e re-interpretadas. Por outro lado, o urbano na perspectiva da Geografia Cultural contribui para o conhecimento da urbanização e da cidade, enfatizando os múltiplos significados que os diversos grupos sociais estabeleceram a respeito dela.

Os estudos sobre o urbano, particularmente sobre a cidade, também estão impregnados de metáforas, metonímias e sinédoques, revelando que a cidade está sendo interpretada como um texto no qual a identidade de uma cidade, vista como lugar dotado de uma singularidade que o distingue dos outros, pode ser de modo marcante o resultado de construção intelectual, derivada de uma tradição étnica cujos traços são permanentemente recriados.

Estas cidades desempenham esse papel de transformação cultural em ampla escala espacial por intermédio de quatro grupos sociais que nelas estão presentes:

Os empresários e executivos das empresas transnacionais, que trabalham em atividade, os imigrantes provenientes dos denominados países do Terceiro Mundo, asiáticos, africanos, latino-americanos e caribenhos, que compõem parcela significativa dessas cidades globais, a exemplo de New York, Londres e Paris, pessoas diretamente vinculadas às atividades culturais strictu sensu e Turistas nacionais e do exterior, que têm vínculo muito transitório com essas cidades globais no qual estas quatro categorias de pessoas estão articuladas entre si, seja no que diz respeito ao mercado de trabalho, seja no que se refere à esfera do consumo . As relações entre cidades globais da cultura e seus espaços são, assim, complexas. Essas cidades constituem focos de criação e de re-interpretação de significados e de formas simbólicas. Aponte-se que esse papel de transformação cultural está profundamente inserido no processo de acumulação de capital, sendo uma de suas expressões.

\section{Bibliografia}

AGNEW, J. MERCER, D. e SOPHER, D. (org.) (1984) - The City in Cultural Context. Londres, Allen \& Unwin. 
AREOLA, D. (1994) - San Antonio - A Mexican American Capital. In: Re-Reading Cultural Geography. Org. K. Foote, P. Hugill, K. Mathewson e J. Smith. Austin, University of Texas Press.

BARNES, T. (1992) - Reading the Texts of Theoretical Economic Geography. The Roles of Physical and Biological Metaphors In: Writing Worlds - Discourse, Texts and Landscape. Org. T. Barnes e J. Duncan. Londres, Routledge.

BERDOULAY, V. (1982) - La Metaphor Organiciste. Contribution à L'Étude du Langage des Géographes. Annales de Géographie, 91, pp. 578-586.

CASTELLS, M. (1989) - The Informational City. Oxford, Basil Blackwell.

CORRÊA, R. L. (2003) - A Geografia Cultural e o Urbano. In: Introdução à Geografia Cultural. Org. R. L. Corrêa e Z. Rosendahl. Rio de Janeiro, Bertrand Brasil.

COSGROVE, D. (1984) - Social Formation and Symbolic Landscape. Londres, Croom Helm. COSGROVE, D. (2000) - Mundos de Significados: Geografia Cultural e Imaginação. In. Geografia Cultural: Um Século (2). Org. R. L. Corrêa e Z. Rosendahl. Rio de Janeiro, EDUERJ. COSTA, A. A. (2000) - A Verticalização e as Transformações do Espaço de Natal (RN). Tese de Doutorado. Departamento de Geografia, UFRJ.

DANIELS, S. e COSGROVE, D. (1993) - Spetacle and Text. Landscape Metaphors in Cultural Geography. In Place/Culture/Representation. Orgs. J. S. Duncan e D. Ley. Londres, Routledge. DOMOSH, M. (1994) - The Symbolism of Skycraper: Case Studies of New York's First Tall Buildings. In Re-Reading Cultural Geography. Org. K. Foote, P. Hugill, K. Matewson e J. Smith. Austin, University of Texas Press.

DUNCAN, J. (1990) - The City as text. Tho Politics of Landscape Intepretation in the Kandyan Kingdom. Cambridge, Cambridge University Press.

DUNCAN, J. (1996) - Me(trope)olis: or Hayden White Among Urbanists. In: Re-Presenting the City - Ethnicity, Captal and the Culture in the Twenty-First Century Metropolis. Org. A. D. King. London, Mamillan.

DUNCAN, J. (2004) - A Paisagem como um Sistema de Criação de Signos. On: Paisagem, Textos e Identidade. Org. R. L. Corrêa e Z. Rosendahl. Rio de Janeiro, EDUERJ.

HANNERZ, U. (1996) - Transnational Connections. Londres, Routledge.

JACKSON, P. (1989) - Maps of Meaning. Londres, Routledge.

LEY, D. (1996). - Urban Geography and Cultural Studies. Urban Geography, 17 (6) pp. 475 477. 
LIVINGSTONE, D. e HARRISON, R. (1982) - Meaning Through Metaphor: Analogy as Epistemology. Annals of the Association of American Geographers, 72 (1), pp. 95-107.

MEINIG, D. (1979) - Symbolic Landscape: Some Idealization of American Communities. In: The Interpretation of Ordinary Landscape. Oxford, Oxford University Press.

NIJMAN, J. (1999) - Cultural Globalization and the Identity of Place: The Reconstruction of Amsterdam. Ecumene, 6 (2), pp. 146-164.

OLIVER, M. (1996) - Historical Preservation and Identity. The Alamo and the Production of a Consumer Landscape. Antipode, 28 (1), 1-23.

RAMIRES, J. C. L. (1998) - A Verticalização do Espaço Urbano de Uberlândia. Uma Análise da Produção e Consumo da Habitação. Tese de Doutorado. Departamento de Geografia, USP. RELPH, E. (1976) - Place ans Placelessness. Londres, Pion.

RELPH, E. (1987) - The Modern Urban Londscape. Londres, Croom Helm.

SILVA, J. M. (2002) - A Verticalização de Guarapuava (PR) e Suas Representações Sociais. Tese de Doutorado. Departamento de Geografia, UFRJ.

SILVA, J. M. (2004) - A Forma Urbana Vertical e Suas Representações Sociais. Espaço e Cultura, 17-18, pp. 17-31.

SMITH, J. M. (1996) - Geographical Rhetoric: Modes and Tropes of Appeal. Annals of the Association of American Geographers, 86 (1), pp. 1-20.

SOUZA, M. A. A. (1994) - A Identidade da Metrópole. São Paulo. HUCITEC/EDUSP.

SPÓSITO, M. E. B. (1991) - “O Chão que Arranha o Céu”. Tese de Doutorado. Departamento e Geografia, USP.

WHEELER, J. (1998) - Urban Cultural Geography - Country Cousin Comes to the City. Urban Geography, 19 (7), pp. 585-590.

WHEELER, J. (2002) - From Urban Economic to Social/Cultural Urban Geography. Urban Geography, 23 (2), pp. 97-102. 